\title{
vCourse: A Course Platform Based on Virtualization Technology
}

\author{
Yu Liu \\ National Experimental Teaching Demonstration Center \\ CSE Department, Beihang University \\ Beijing, China \\ buaa_liuyu@buaa.edu.cn
}

\author{
Jinmo Wang \\ School of Computer Science and Technology \\ Beihang University \\ Beijing, China \\ jinmo_wang@163.com
}

\begin{abstract}
This paper presents a platform named vCourse based on the virtualization technology. vCourse supports multiple operation systems and multiple courses online. Teachers can create a course template and deploy it as many virtual machines. Students can do their exercises on those virtual machines and interact with others and the teacher. The features, structure and key mechanisms of vCourse are discussed in this paper. Two experiments are designed to evaluate the performance of vCourse. According to the test results, vCourse can run the typical software smoothly and is not very sensitive on the virtual machine numbers.
\end{abstract}

Keywords- virtualization technology; virtual classroom; distance education; experiment education

\section{INTRODUCTION}

High quality advanced technical education is a popular approach to produce well-educated graduates who can contribute to the development of new generations of technology[1]. Laboratory experiences play a central role in an advanced technical education[2]. During the experiences, students can put the concept into practice and get a more intuitive understanding of the technologies. They also have the potential for increasing the synergy between research and education[3]. In computer science and engineering or related education areas, there some challenges in laboratory experiences. Firstly, the courses and experiences need many high performance hardware platforms. Secondly, it is hard work for test administrators to maintain so many platforms which need different software environment respectively. At the same time, the distance education platforms for experiences play a more importance role in high quality advanced technical education[4].

In this paper, we will propose a course platform for software related information courses education base on virtualization technology[5][6], and our platform is name as vCourse. In vCourse, in order to share and reuse the limited hardware resources of the computers, we separate and encapsulate the hardware by the virtualization methods. All the resources can be dynamic allocate because they are managed by the virtual machine and virtual network technologies. In addition, vCourse supports virtual machine migration and snapshot features, with which the experiment environment can be real-time saved and replayed. The virtual machine produced by vCourse is transparent and segregate. All the virtual machines run separately. vCourse is a multicourse supported platform which can save hardware costs.

The main advantages of vCourse are:
- Support multiple operation systems and multiple courses.

- Support course template(snapshot), teachers can make a template of their courses and give it to all the students.

- $\quad$ Support cooperation between teachers and students. Teachers can share his screen to all or scratch either student's screen.

- Distance education. Teachers and students can remote login vCourse platform and do the experiments as if it is on the local machines.

\section{THE DESIGN OF VCOURSE}

vCourse is designed and implemented based on virtualization technology. In vCourse, the users can create VM-based course environments in only a few minute, which greatly reduce the cost for creating and maintaining teaching and experimenting environments. vCourse leverage a set of advanced virtualization techniques such as virtual machine migration, virtual machine snapshot, to facilitate the teaching and learning activities for both teachers and students.

In this chapter, we will first introduce the basic functions of vCourse, then propose the design and architecture of vCourse, and show the implementation of vCourse at the end.

\section{A. Core Functions for Teachers}

On vCourse, teachers can do following operations:

- Remote login. Teachers can login the platform via remote clients. In ones' own teaching environment, the teacher can arrange and check the homework.

- Course template design. vCourse provides teachers with the ability to customize their own course environments. For example, a teacher can create a virtual machine template which contains an operating system and some proper software applications for teaching. The template can be duplicated to create multiple virtual machine instances which can serve as the course environments for each student.

- Course and experiment representation. The demonstration is very important for the experiment course. vCourse supports screen transport, students can see the teachers' screen synchronously. At the same time, the voice and video of the teacher will be played on the students' clients. vCourse also supports 
the feature of screen record based on the VNC technology[7].

\section{B. Core Functions for Students}

For the students, vCourse is a learning platform which provide the following features:

- Distance learning. Students can access the platform via network from the remote clients and do some learning operations such as course trace, homework, online test, test result query and course feedback. And they can do all of these works in anytime, from anywhere that has an internet environment..

- Virtual classroom. When logged in the vCourse platform, there is a virtual classroom. A Student can study online and interaction with others or the teacher. The teacher online can enter the student virtual machine and give him some operation guide if he needed. This feature can help the student to learn more quickly and convenient.

- Course management. If a student can not finish his practice in one time, he can save the task process of the virtual machine in vCourse. With the course snapshot function, students can save or reopen a course process at any time. For a reopened excise, all the status are resumed and the student can go on the experiment. The student can also make a copy for the current virtual machine. In order to cooperate with other, a student can send his copy to others or to the teacher.

\section{The Software Architecture of vCourse}

vCourse platform has two important components, the Designer and the Manager. The Designer is a function set used by the teacher and administrator. By this component, the user can create the virtual machine and the course template which is the seed of the course. The Manager component is used by the teacher and the student. They can access and manage the course instance by the Manager component.

Fig. 1 shows the main architecture of vCourse platform. In the Designer component, there are three main models, which are:

- Snapshot Tools. This module is used to manage the remote or local storage resources.

- VM Factory. It is a factory to produce the virtual machine. This module is running on Linux OS and all the virtual instances are stored in it. It also has a serial of interfaces to operate the virtual instance, such as the generate interface, the modify interface and the delete interface.

- VNet Factory. It is another factory to create the virtual network instance and it is also running on the Linux OS. The network instances and related configurations include virtual network drivers, virtual switches and virtual routers are stored in this module.

The Manager component proposes the online access and virtual machine management functions. It includes:
- Virtual Resource Directory. This module supports the discovery and search functions for online virtual resources. Before create a virtual machine, the user should get the service information from the sever by the this module and then create the virtual machine on the physical computer according to that information.

- VM Container. This module is a container for all the virtual machines. Users who want to share their computing resources should firstly install this module. It is running on Linux and is responsible for the management of the virtual machines. The virtual operations such as start, stop, suspend, resume and remote deployment are all supported by it. It can also be separated on the functional aspect into two layer, which are the Hypervisor Layer and the Remote Control Layer.

- vDesktop. It is the user operation interface of vCourse. Users logged in the platform can use this module to access all the virtual resources and services. vDesktop is built on the Web technology and can run on either Windows or Linux systems. Dynamic View Static View

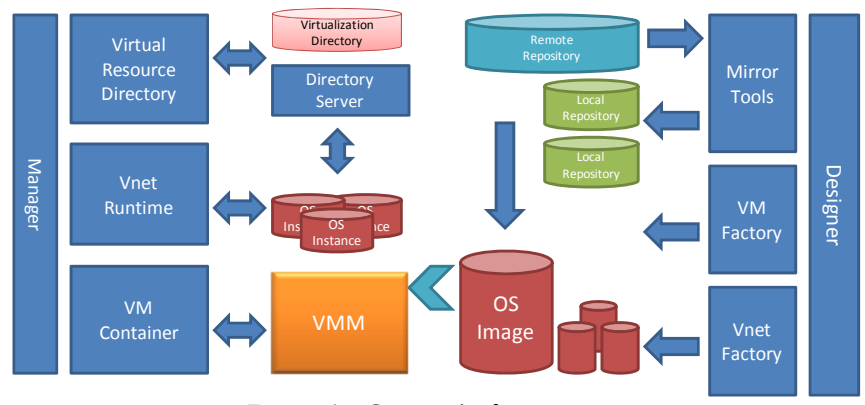

Figure 1. vCourse Architecture

\section{PLATFORM IMPLEMENTATION}

VM Container and VM Factory are the key modules of vCourse, then we will show the implementation details of the two modules.

\section{A. VM Container}

VM Container will timed report its running status to Virtual Resource Directory and vDesktop. The status include:

- CPU, memory, disk, network status of the physical machine.

- CPU, memory, disk, network status of the virtual machine.

The status monitor mechanism is based on the Push model. The machines under observation will actively send their status to the monitor. We use two communication mechanisms:

- REST interface on vDesktop server. It can be accessed via https. The VM Container will post the current status to the server.

- Installing Collectd Server on vDesktop server and Collectd Client on the VM Container. Then the status can be transferred via Collectd.. 


\section{B. VM Factory}

VM Factory supports mulitiple VM deployment methods for VM Container. They are:

- $\quad$ Download the VM mirror or SDK from VM Factory. vCourse supports this downloading by Http or NFC.

- Mount the virtual dist of the VM Factory server by NFS.

- $\quad$ Load the ISO image on VM Factory server and do a CD installation.

- $\quad$ Online start by the TFTP server on the VM Factory server.

\section{Advanced Functions}

To enable the backup and recovery of course VMs, We develop some advanced techniques to support live VM snapshot and rollback. We develop a live VM snapshot and rollback mechanism which can save the running state of Virtual Machines at set intervals without disturb the normal execution of VMs. If we encounter the crash or failure of a course VM. We can resume to the saved checkpoints and continue our course. We also develop a VM screen copying technique based on TCP multicast mechanism, which can duplicate teacher's course VM desktop to every student in the class.

\section{Security Considerations}

vCourse supports user-remote-access, so the user and system security is critical to vCourse. VCourse fully considers the security needs of users, providing security roles and access control permissions for users.

Username and password authentication. Before using VM Container and VM Factory by vDesktop, the users need to enter username and password for authentication.

After pass the authentication of vDesktop, the users will get its own RSA public key, and implement hierarchical rights management.

- Guest can run a read-only command and browse the course list, or apply to be a user.

- Common users can access and modify the virtual machine instances, start, stop and access the virtual machine belongs to own.

- Advanced user can create a virtual machine templates, deploy virtual machine instances, access and modify the instances, start, stop and access the virtual machine belongs to own.

- Administrator can approved guest as the user, modify all virtual machine templates, mirror, start and stop all virtual machines.

Teacher and student are granted corresponding security role by the administrator. The role of the teacher is normally advanced users, and the student is a common user.

\section{E. The Choice of VMM}

Hypervisor is a virtual machine monitor(VMM). The virtual machine container includes the Hypervisor layer, which providing operation support for virtual machine instances. vCourse does not reinvent the wheel to realize virtual machine, but just directly use the available virtual machine technology. Including:

- KVM. A type of virtual machine which develops quickly now. KVM supports the online migration, realize load equalization and adaptive. It can run Windows directly based on Intel VT chip. And it is a type of virtual machine that vCourse mainly supports.

- Chroot. The function of Chroot is very limited. It only provides file isolation but can not virtualize hardware. It also has not CPU, memory and virtual network card. So the network is not supported. But the installation is convenient, so it is another major type virtual machine that vCourse supports.

- OpenVZ. It is a type of virtual machine which can partly enter into the Linux kernel of 2.6.19, also supports the online migration. OpenVZ runs Linux only, but can be nested into VMWare.

- VServer. A virtual machine officially supported by Debian, which similar to OpenVZ and only runs on Linux. It can be nested into VMWare.

- User-mode-linux. A virtual machine which can enter the Linux kernel of 2.6.9, low efficiency, but easy to install.

\section{F. Configuration of vCourse}

All the modules in vCourse are designed to automatic configuration. The users can easily use after download without complicated configuration process. For example, the users who want to share the computing resource can release their computing resources in the LAN after installing VM Container and without configuration. For the users who want to use the virtual computing resources can find the virtual computing resources in the LAN by the Virtual Resource Directory without configuring the directory server address.

\section{PERFORMANCE EXPERIMENT}

In order to test the efficiency of vCourse. We designed two experiments. And we choose KVM as the VMM in our test.

In the first experiment, we deployed five typical software in computer science education to evaluate the performance of a single virtual machine in vCourse. The five test objects are Photoshop, CUP 2006, Quartus II, MS VS2010 and MS SqlServer 2005. According to the test result is shown in Fig.2, the performance loss of the VM for Photoshop and Quartus II are about 15\%, for MS SqlServer2005 is about $10 \%$, and for CPU2006 is less than $5 \%$. This means a student can use these software on a vCourse virtual machine and get almost the same user experience on a physical machine.

In the second experiment, we test six typical operations in vCourse with different numbers of VMs. Table.1 shows the result and we can see that all the operations need less than 1 minute except the Deploy operation for 16 Nodes. But the Deploy can be done by the teacher at any free time and it is not a time critical operation related with the course process. We can also know that with the increasing of VM, the 
performance of vCourse will drop down a little, so our platform is not very sensitive on VM numbers.

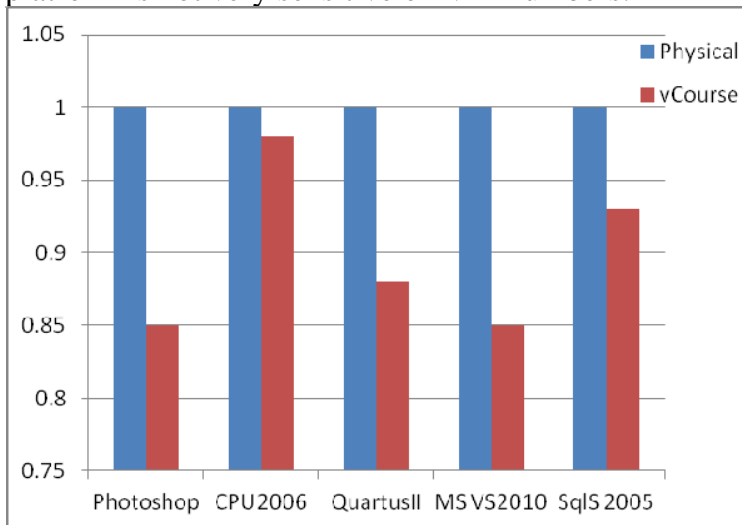

Figure 2 Performance test for a single VM

TABLE I. EXECUTION TIME FOR TYPICAL OPERATIONS IN VCOURSE

\begin{tabular}{|l|l|c|}
\hline \multirow{2}{*}{ Operations } & \multicolumn{2}{|c|}{ Execution Time (s) } \\
\cline { 2 - 3 } & \multicolumn{1}{|c|}{ 8 Nodes } & 16 Nodes \\
\hline Deploy & 50 & 93 \\
\hline Start & 12 & 16 \\
\hline Stop & 1 & 1 \\
\hline Resume & 1 & 2 \\
\hline Snapshot & 12 & 14 \\
\hline Rollback & 12 & 15 \\
\hline
\end{tabular}

\section{CONCLUSION AND FUTURE WORK}

In this paper, we propose the vCourse which is a virtualized Teaching and Experimenting Platform for hosting multiple courses. We also present the design of vCourse and a prototype of vCourse is implemented based on virtual machine and virtual networking technologies. vCourse can help both teachers and students to access and manage their course environments, and improve the efficiency of teaching and learning. To verify the effectiveness and efficiency of
vCourse, we conduct several comprehensive experiments. And the results show that vCourse can manage the underlying physical resources in an efficiently way and can successfully host multiple courses without no mutual interference. Furthermore, vCourse can provide online users with teaching resources in a remote and convenient way. Other users can leverage vCourse to customize their own teaching course without the need to buy computers and build their own experimental environments.

\section{ACKNOWLEDGMENT}

The authors would like to thank all members of the National Experimental Teaching Demonstration Center at Beihang University for their comments and fruitful discussions during the past years that made this work possible. This work has been supported by the National Natural Science Funds of China(61202238).

\section{REFERENCES}

[1] Lynch, R. L., "High school career and technical education for the first decade in the 21st century, " J. Vocational Education Research, vol.25(2), May 2000, pp.155-198.

[2] Edward, N. S., "The role of laboratory work in engineering education: Student and staff perceptions, ” Int. J. Electrical Eng. vol. 39, 2002, pp.11-19.

[3] Squires D.,Preece J., "Predicting quality in educational software: evaluating for learning, usability, and the synergy between them, "Int J. Comput, vol.11,May 1999,pp.467-483.

[4] Sherry M. Cummingsa,Leonora Foelsb, Kate M. Chaffina, "Comparative Analysis of Distance Education and Classroom-based Formats for a Clinical Social Work Practice Course, ” Int. J. Social Work Education, vol 32, Jan 2013, pp.68-80.

[5] A. A. Semnanian, J. Pham, B. Englert, and X. Wu, "Virtualization technology and its impact on computer hardware architecture," in Information Technology: New Generations (ITNG), 2011 Eighth International Conference on, Apr 2011, pp. 719-724.

[6] L. Wang, G. V. Laszewski, M. Kunze, and J. Tao. Cloud Computing: a Perspective Study. Proceedings of the Grid Computing Environments (GCE) workshop, November 2008.

[7] M. Wannous and H. Nakano, "NVLab, A Networking Virtual WebBased Laboratory that Implements Virtualization and Virtual Network Computing Technologies," IEEE Transactions on Learning Technologies, vol. 3, Apr/Jun 2010, pp. 129-138. 